Edith Cowan University

Research Online

ECU Publications 2013

$1-1-2013$

\title{
Association of hematological parameters with metabolic syndrome in Beijing adult population: a longitudinal study
}

Li-Xin Tao

Xia Li

Hui-Ping Zhu

Da Huo

Tao Zhou

See next page for additional authors

Follow this and additional works at: https://ro.ecu.edu.au/ecuworks2013

Part of the Medicine and Health Sciences Commons

10.1007/s12020-013-0067-z

This is an Author's Accepted Manuscript of: Tao, L., Li, X., Zhu, H., Huo, D., Zhou, T., Pan, L., Luo, Y., Wang, W. , Wang, Z., Chen, D., Wu , L., \& Guo, X. (2013). Association of hematological parameters with metabolic syndrome in Beijing adult population: a longitudinal study. Endocrine, 46(3), 485-495. The final publication is available at Springer via here

This Journal Article is posted at Research Online.

https://ro.ecu.edu.au/ecuworks2013/781 


\section{Authors}

Li-Xin Tao, Xia Li, Hui-Ping Zhu, Da Huo, Tao Zhou, Lei Pan, Yan-Xia Luo, Wei Wang, Zhao-Ping Wang, Dong-Ning Chen, Li-Juan Wu, and Xiu-Hua Guo 
Dear Author,

\section{Springer}

Here are the proofs of your article.

- You can submit your corrections online, via e-mail or by fax.

- For online submission please insert your corrections in the online correction form. Always indicate the line number to which the correction refers.

- You can also insert your corrections in the proof PDF and email the annotated PDF.

- For fax submission, please ensure that your corrections are clearly legible. Use a fine black pen and write the correction in the margin, not too close to the edge of the page.

- Remember to note the journal title, article number, and your name when sending your response via e-mail or fax.

- Check the metadata sheet to make sure that the header information, especially author names and the corresponding affiliations are correctly shown.

- Check the questions that may have arisen during copy editing and insert your answers/ corrections.

- Check that the text is complete and that all figures, tables and their legends are included. Also check the accuracy of special characters, equations, and electronic supplementary material if applicable. If necessary refer to the Edited manuscript.

- The publication of inaccurate data such as dosages and units can have serious consequences. Please take particular care that all such details are correct.

- Please do not make changes that involve only matters of style. We have generally introduced forms that follow the journal's style.

Substantial changes in content, e.g., new results, corrected values, title and authorship are not allowed without the approval of the responsible editor. In such a case, please contact the Editorial Office and return his/her consent together with the proof.

- If we do not receive your corrections within $\mathbf{4 8}$ hours, we will send you a reminder.

- Your article will be published Online First approximately one week after receipt of your corrected proofs. This is the official first publication citable with the DOI. Further changes are, therefore, not possible.

- The printed version will follow in a forthcoming issue.

\section{Please note}

After online publication, subscribers (personal/institutional) to this journal will have access to the complete article via the DOI using the URL: http://dx.doi.org/[DOI].

If you would like to know when your article has been published online, take advantage of our free alert service. For registration and further information go to: http://www.springerlink.com.

Due to the electronic nature of the procedure, the manuscript and the original figures will only be returned to you on special request. When you return your corrections, please inform us if you would like to have these documents returned. 


\section{Metadata of the article that will be visualized in OnlineFirst}

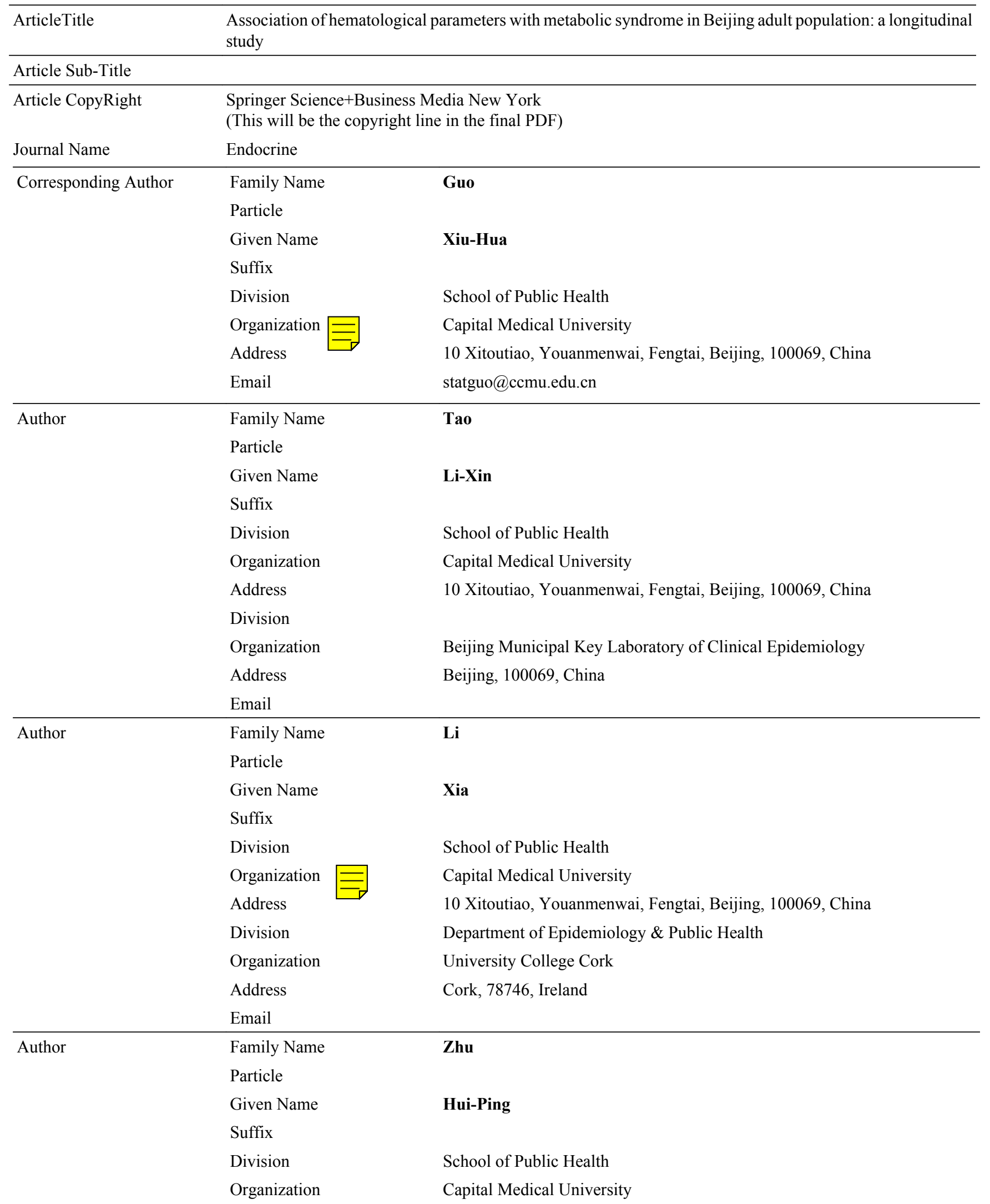


Address

10 Xitoutiao, Youanmenwai, Fengtai, Beijing, 100069, China

Division

Organization

Beijing Municipal Key Laboratory of Clinical Epidemiology

Address

Beijing, 100069, China

Email

\begin{tabular}{|c|c|c|}
\hline \multirow[t]{8}{*}{ Author } & Family Name & Huo \\
\hline & Particle & \\
\hline & Given Name & Da \\
\hline & Suffix & \\
\hline & Division & Institute for Infectious Disease and Endemic Disease Control \\
\hline & Organization & Beijing Center for Disease Prevention and Control \\
\hline & Address & Beijing, 100013, China \\
\hline & Email & \\
\hline \multirow[t]{11}{*}{ Author } & Family Name & Zhou \\
\hline & Particle & \\
\hline & Given Name & Tao \\
\hline & Suffix & \\
\hline & Division & School of Public Health \\
\hline & Organization & Capital Medical University \\
\hline & Address & 10 Xitoutiao, Youanmenwai, Fengtai, Beijing, 100069, China \\
\hline & Division & \\
\hline & Organization & Beijing Municipal Key Laboratory of Clinical Epidemiology \\
\hline & Address & Beijing, 100069, China \\
\hline & Email & \\
\hline \multirow[t]{11}{*}{ Author } & Family Name & Pan \\
\hline & Particle & \\
\hline & Given Name & Lei \\
\hline & Suffix & \\
\hline & Division & School of Public Health \\
\hline & Organization & Capital Medical University \\
\hline & Address & 10 Xitoutiao, Youanmenwai, Fengtai, Beijing, 100069, China \\
\hline & Division & \\
\hline & Organization & Beijing Municipal Key Laboratory of Clinical Epidemiology \\
\hline & Address & Beijing, 100069, China \\
\hline & Email & \\
\hline \multirow[t]{10}{*}{ Author } & Family Name & Luo \\
\hline & Particle & \\
\hline & Given Name & Yan-Xia \\
\hline & Suffix & \\
\hline & Division & School of Public Health \\
\hline & Organization & Capital Medical University \\
\hline & Address & 10 Xitoutiao, Youanmenwai, Fengtai, Beijing, 100069, China \\
\hline & Division & \\
\hline & Organization & Beijing Municipal Key Laboratory of Clinical Epidemiology \\
\hline & Address & Beijing, 100069, China \\
\hline
\end{tabular}


Email

\begin{tabular}{|c|c|c|}
\hline \multirow[t]{11}{*}{ Author } & Family Name & Wang \\
\hline & Particle & \\
\hline & Given Name & Wei \\
\hline & Suffix & \\
\hline & Division & School of Public Health \\
\hline & Organization $\equiv$ & Capital Medical University \\
\hline & Address & 10 Xitoutiao, Youanmenwai, Fengtai, Beijing, 100069, China \\
\hline & Division & School of Medical Science \\
\hline & Organization & Edith Cowan University \\
\hline & Address & Mt Lawley, WA, 6050, Australia \\
\hline & Email & \\
\hline \multirow[t]{8}{*}{ Author } & Family Name & Wang \\
\hline & Particle & \\
\hline & Given Name & Zhao-Ping \\
\hline & Suffix & \\
\hline & Division & Department of Physical Examination \\
\hline & Organization & Beijing Tongren Hospital \\
\hline & Address & Beijing, 100041, China \\
\hline & Email & \\
\hline \multirow[t]{8}{*}{ Author } & Family Name & Chen \\
\hline & Particle & \\
\hline & Given Name & Dong-Ning \\
\hline & Suffix & \\
\hline & Division & Department of Physical Examination \\
\hline & Organization & Beijing Tongren Hospital \\
\hline & Address & Beijing, 100041, China \\
\hline & Email & \\
\hline \multirow[t]{11}{*}{ Author } & Family Name & $\mathbf{W u}$ \\
\hline & Particle & \\
\hline & Given Name & Li-Juan \\
\hline & Suffix & \\
\hline & Division & School of Public Health \\
\hline & Organization & Capital Medical University \\
\hline & Address & 10 Xitoutiao, Youanmenwai, Fengtai, Beijing, 100069, China \\
\hline & Division & \\
\hline & Organization & Beijing Municipal Key Laboratory of Clinical Epidemiology \\
\hline & Address & Beijing, 100069, China \\
\hline & Email & \\
\hline
\end{tabular}

Received

22 July 2013

Schedule

Revised

Accepted

13 September 2013

Abstract

The purposes of the study were to estimate the incidence of metabolic syndrome (MetS) and to systematically evaluate the relationship between hematological parameters and MetS in a 5-year follow-up of Beijing adult population. The longitudinal study included 3,180 adults, aged 20-65 years, who attended health check-ups 
in Beijing Tongren Hospital in 2007 and 2012. Multivariate logistic regression was conducted to explore the associations between hematological parameters and MetS. The 5-year cumulative incidence of MetS in this sample was $10.82 \%$ (14.22\% for males and $7.59 \%$ for females). Among all the hematological parameters, white blood cell count (WBC) was positively associated with MetS for 20-35-year-old (male OR 1.482, $95 \%$ CI 1.169-2.974; female OR 1.398, $95 \%$ CI 1.145-3.011), and 36-50-year-old (male OR 2.012, $95 \%$ CI 1.290-4.010; female OR 3.400, $95 \%$ CI 1.818-4.528) male and female subjects. Alanine aminotransferase (ALT) was significantly associated with the incidence of MetS for males (20-35-year-old OR 2.080, $95 \%$ CI 1.371-3.159; 36-50-year-old OR 2.421, 95 \% CI 1.335-3.412; 51-65-year-old OR 4.267, 95 \% CI 1.1616.781). Low-density lipoprotein cholesterol (LDL-C) was positively associated with MetS for 51-65-yearold (male OR 3.078, $95 \%$ CI 2.468-5.131; female OR 2.140, $95 \%$ CI 1.524-4.359) for male and female subjects. WBC is positively associated with MetS for young adults, while LDL-C is positively associated with MetS for elderly people. ALT is positively associated with MetS for males. Our findings provide further evidence in support of using hematological markers for early detection of individuals at risk for MetS.

Keywords (separated by '-') Hematological parameters - Metabolic syndrome - Association - Longitudinal study

Footnote Information 


\title{
2 Association of hematological parameters with metabolic syndrome 3 in Beijing adult population: a longitudinal study
}

\author{
4 Li-Xin Tao $\cdot$ Xia Li $\cdot$ Hui-Ping Zhu $\cdot$ Da Huo $\cdot$ Tao Zhou $\cdot$ Lei Pan \\ 5 Yan-Xia Luo $\cdot$ Wei Wang $\cdot$ Zhao-Ping Wang $\cdot$ Dong-Ning Chen • \\ 6 Li-Juan Wu $\cdot$ Xiu-Hua Guo
}

7 Received: 22 July 2013/Accepted: 13 September 2013

8 (C) Springer Science+Business Media New York 2013

L.-X. Tao · X. Li · H.-P. Zhu · T. Zhou · L. Pan · Y.-X. Luo W. Wang $\cdot$ L.-J. Wu $\cdot$ X.-H. Guo $(\square)$

School of Public Health, Capital Medical University, 10

Xitoutiao, Youanmenwai, Fengtai, Beijing 100069, China

e-mail: statguo@ccmu.edu.cn

L.-X. Tao - H.-P. Zhu - T. Zhou - L. Pan · Y.-X. Luo - L.-J. Wu Beijing Municipal Key Laboratory of Clinical Epidemiology,

Beijing 100069, China

X. Li

Department of Epidemiology \& Public Health, University College Cork, Cork 78746, Ireland

\section{Huo}

Institute for Infectious Disease and Endemic Disease Control, Beijing Center for Disease Prevention and Control, Beijing 100013, China

W. Wang

School of Medical Science, Edith Cowan University, Mt Lawley, WA 6050, Australia

\section{Z.-P. Wang · D.-N. Chen}

Department of Physical Examination, Beijing Tongren Hospital, Beijing 100041, China associated with MetS for 20-35-year-old (male OR 1.482, $95 \%$ CI 1.169-2.974; female OR 1.398, $95 \%$ CI 1.145-3.011), and 36-50-year-old (male OR 2.012, $95 \%$ CI 1.290-4.010; female OR 3.400, $95 \%$ CI 1.818-4.528) male and female subjects. Alanine aminotransferase (ALT) was significantly associated with the incidence of MetS for males (20-35-year-old OR 2.080, $95 \%$ CI 1.371-3.159; 36-50year-old OR 2.421, $95 \%$ CI 1.335-3.412; 51-65-year-old OR 4.267, $95 \%$ CI 1.161-6.781). Low-density lipoprotein cholesterol (LDL-C) was positively associated with MetS for 51-65-year-old (male OR 3.078, $95 \%$ CI 2.468-5.131; female OR 2.140, $95 \%$ CI 1.524-4.359) for male and female subjects. WBC is positively associated with MetS for young adults, while LDL-C is positively associated with MetS for elderly people. ALT is positively associated with MetS for males. Our findings provide further evidence in support of using hematological markers for early detection of individuals at risk for MetS.

Keywords Hematological parameters - Metabolic syndrome $\cdot$ Association $\cdot$ Longitudinal study

\section{Introduction}

Metabolic syndrome (MetS) is a cluster of risk factors that include abdominal obesity, hyperglycemia, raised blood pressure (BP), low high-density lipoprotein cholesterol (HDL-C), and high triglycerides (TG). Since prevalence of MetS is rapidly growing and it is associated with an increased risk of insulin resistance, diabetes, cardiovascular disease (CVD), and total mortality [1-3], the identification for biomarkers of MetS is of pivotal importance.

The prevalence of MetS increased with age for both sexes [4]. Several non-inflammatory biomarkers have been

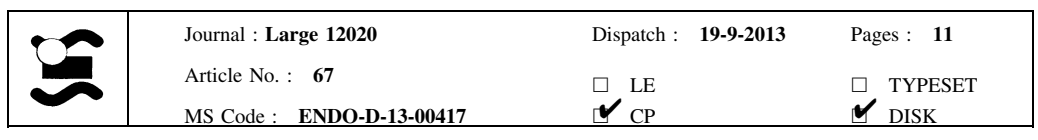


associated with MetS and its components in different populations, including growth factors [5], micro albuminuria [6], and uric acid (UA) [7, 8]. Elevated white blood cell (WBC) count is intimately linked to the prevalence and future development of MetS in populations of working subjects $[9,10]$. Insulin resistance and/or hyperinsulinemia have been shown to correlate with WBC counts [11]. Elevated liver enzymes, especially alanine aminotransferase (ALT) may be related with and a better predictor for MetS [12-14]. Other hematological parameters including platelet counts (PLT), hemoglobin (HGB), hematocrit concentrations (HCT), C-reactive protein, and serum bilirubin increased with increasing numbers of MetS components [15-18]. To the best of our knowledge, there have been few studies conducting systematic evaluation for relationship between hematological parameters and MetS for different age groups of males and females in a large Beijing adult population.

Therefore, the aims of this study were to estimate the incidence of MetS and to investigate prospective associations between blood parameters and MetS in a Beijing adult population.

\section{Materials and methods}

Subjects

A total of 3,832 subjects aged 20-65 years who attended health check-ups in Beijing Tongren Hospital, China, in 2007 and 2012 were enrolled in the study. Individuals with a previous diagnosis of CVD, cerebral infarction or gastric cancer, or those who had undergone coronary artery bypass surgery, coronary stenting surgery or gastrectomy, or those who had MetS at baseline were excluded. The remaining 3,180 subjects were included in the final analysis. The study was approved by the Ethics Committee of Capital Medical University (approval number: 2013SY26). Written informed consent was obtained from all the participating subjects.

\section{Measurements}

Information about medication use was gathered by trained medical staff during a standardized interview. Subjects who reported taking anti-hypertensive, anti-dyslipidemic, or anti-diabetic drugs were considered to have elevated BP, elevated TG, reduced HDL-C, or elevated fasting plasma glucose (FPG).

The participants underwent routine physical examinations that included the measurement of height, weight, BP, and overnight fasting blood sampling. Weight and height were measured without shoes, and body mass index (BMI) was calculated as weight $(\mathrm{kg})$ divided by squared height (m). BP was measured on the right arm of subjects seated and at rest for at least 5 min by a trained nurse. During the 30 min preceding the measurements, the subjects were required to refrain from smoking or consuming caffeine. A standard mercury sphygmomanometer was used with one of four cuff sizes (pediatric, regular adult, large adult, or thigh) based on the participant's arm circumference. Three readings each of systolic and diastolic BPs were recorded, with an interval of $1 \mathrm{~min}$ at least, and the average of the last two measurements was used for data analysis.

Blood samples were obtained from antecubital vein into tubes containing EDTA in the morning after an overnight fasting period. Red blood cell (RBC), WBC, lymphocyte, neutrophil, mean corpuscular hemoglobin $(\mathrm{MCH})$, PLT, mean platelet volume (MPV), platelet distribution width (PDW), and HGB were measured by an autoanalyzer (Sysmex SE-9000, Kobe, Japan). HDL-C, TG, FPG, ALT, aspartate aminotransferase (AST), UA, and LDL-C were measured by enzymatic method using a chemistry analyzer (Beckman LX 20, USA) at the central laboratory of the hospital. All analyses were performed in accordance with the manufacturer's recommendations.

\section{Definitions}

MetS was diagnosed if the subjects had three or more risk determinants according to the Joint Interim Statement criteria [19]. However, in this study, waist circumference (WC) was not measured because of limited health check-up site, and BMI was taken as a substitute for the component of obesity [20]. The determinants were as follows:

(1) Obesity: BMI $\geq 28 \mathrm{~kg} / \mathrm{m}^{2}$. (2) Elevated TG (drug treatment for elevated $\mathrm{TG}$ is an alternate indicator): $\geq 150 \mathrm{mg} / \mathrm{dL}$ (1.7 mmol/L). (3) Reduced HDL-C (drug treatment for reduced HDL-C is an alternate indicator): $<40 \mathrm{mg} / \mathrm{dL}(1.0 \mathrm{mmol} / \mathrm{L})$ in males, $<50 \mathrm{mg} / \mathrm{dL}$ ( $1.3 \mathrm{mmol} /$ L) in females. (4) Elevated BP (antihypertensive drug treatment in a patient with a history of hypertension is an alternate indicator): systolic $\geq 130 \mathrm{mmHg}$ and/or diastolic $\geq 85 \mathrm{mmHg}$, and (5) Elevated FPG (drug treatment of elevated glucose is an alternate indicator): $\geq 100 \mathrm{mg} / \mathrm{dL}$.

Statistical analysis

Data were expressed as mean \pm standard deviation (SD) or, for non-normally distributed variables, as median and interquartile range. To compare the differences between groups, student's $t$ test or Wilcoxon rank sum test was used for continuous variables, and $\chi^{2}$ test or Fisher's exact test was used for categorical variables. Log transformations were applied to skewed data prior to parametric analyses. Multiple logistic regression analysis was used to assess the

$\begin{array}{lll}\text { Journal : Large 12020 } & \text { Dispatch : 19-9-2013 } & \text { Pages : } \mathbf{1 1} \\ \text { Article No. : } \mathbf{6 7} & \square \text { LE } & \square \text { TYPESET } \\ \text { MS Code : ENDO-D-13-00417 } & \boldsymbol{V}_{\mathrm{CP}} & \checkmark \text { DISK }\end{array}$


relationship between hematological parameters and MetS after adjusting for medication use. Data were analysed using the SAS software package (version 9.2; SAS Institute, Chicago, IL, USA), and $P<0.05$ was considered significant.

\section{Results}

The incidence of MetS and prevalence of its components by age and gender

The sample of this study represents 3,180 subjects, including 1,547 males and 1,633 females aged 20-65 years old. The sex- and age-specific incidence of MetS, prevalence of its components, and medical use are shown in Table 1 and Figs. 1 and 2.

Overall, the 5-year cumulative incidence of MetS among all subjects was $10.82 \%$, with $14.22 \%$ of males and $7.59 \%$ of females having MetS. Of note, the 5-year cumulative incidence of MetS among 36-50-year-old male subjects was the highest $(15.40 \%)$. And the 20-35-yearold female subjects had the lowest incidence $(4.13 \%)$. While among female subjects, the 51-65-year-old subjects had the highest 5-year cumulative incidence of MetS $(14.05 \%)$.

The prevalence of MetS components is shown in Fig. 2. The prevalence of elevated BP and elevated FPG increased with age for male subjects, while the prevalence decreased with age for reduced HDL-C and elevated BMI for male subjects. The highest prevalence of elevated TG was presented in 36-50-year-old group among males. For female

Table 1 The incidence of MetS, prevalence of its components and medication use

\begin{tabular}{|c|c|c|c|c|c|}
\hline \multirow[t]{2}{*}{ Gender } & \multirow[t]{2}{*}{ Variables } & \multicolumn{3}{|c|}{ Age at inclusion (years) } & \multirow[t]{2}{*}{$P$ value } \\
\hline & & $20-35$ & $36-50$ & $51-65$ & \\
\hline \multirow[t]{10}{*}{ Male } & $N$ & 681 & 591 & 275 & \\
\hline & MetS, $n(\%)$ & $94(13.80)$ & $91(15.40)$ & $35(12.73)$ & 0.5296 \\
\hline & Elevated BP, $n(\%)$ & $190(27.90)$ & $202(34.18)$ & $118(42.91)$ & $<0.0001$ \\
\hline & Elevated TG, $n(\%)$ & $216(31.72)$ & $208(35.19)$ & $75(27.27)$ & 0.0623 \\
\hline & Reduced HDL-C, $n(\%)$ & $170(27.96)$ & $111(18.78)$ & $42(15.27)$ & 0.0011 \\
\hline & Elevated BMI, $n(\%)$ & $104(15.27)$ & $75(12.69)$ & $27(9.82)$ & 0.0682 \\
\hline & Elevated FPG, $n(\%)$ & $62(18.36)$ & $203(35.36)$ & $87(46.55)$ & $<0.0001$ \\
\hline & Anti-hypertensive drugs, $n(\%)$ & $5(0.73)$ & $70(11.81)$ & $51(18.55)$ & $<0.0001$ \\
\hline & Anti-dyslipidemic drugs, $n(\%)$ & $41(6.02)$ & $82(13.87)$ & $37(13.45)$ & $<0.0001$ \\
\hline & Anti-diabetic drugs, $n(\%)$ & $31(4.55)$ & $74(12.61)$ & $37(13.45)$ & $<0.0001$ \\
\hline \multirow[t]{10}{*}{ Female } & $N$ & 533 & 858 & 242 & \\
\hline & MetS, $n(\%)$ & $22(4.13)$ & $68(7.93)$ & $34(14.05)$ & 0.0023 \\
\hline & Elevated BP, $n(\%)$ & $42(7.88)$ & $151(17.60)$ & $78(32.23)$ & $<0.0001$ \\
\hline & Elevated TG, $n(\%)$ & $41(7.69)$ & $144(16.78)$ & $52(21.49)$ & $<0.0001$ \\
\hline & Reduced HDL-C, $n(\%)$ & $140(26.27)$ & $211(24.59)$ & $56(23.14)$ & 0.6141 \\
\hline & Elevated BMI, $n(\%)$ & $17(3.19)$ & $52(6.06)$ & $16(6.61)$ & 0.0363 \\
\hline & Elevated FPG, $n(\%)$ & $125(11.63)$ & $209(23.66)$ & $128(35.97)$ & $<0.0001$ \\
\hline & Anti-hypertensive drugs, $n(\%)$ & $6(1.13)$ & $50(5.83)$ & $47(19.42)$ & $<0.0001$ \\
\hline & Anti-dyslipidemic drugs, $n(\%)$ & $2(0.38)$ & $11(1.28)$ & $15(6.20)$ & $<0.0001$ \\
\hline & Anti-diabetic drugs, $n(\%)$ & $16(3.00)$ & $38(4.43)$ & $28(11.57)$ & $<0.0001$ \\
\hline
\end{tabular}

Met $S$ metabolic syndrome, $B P$ blood pressure, $T G$ triglycerides, $H D L-C$ high-density lipoprotein cholesterol, $B M I$ body mass index, $F P G$ fasting plasma glucose

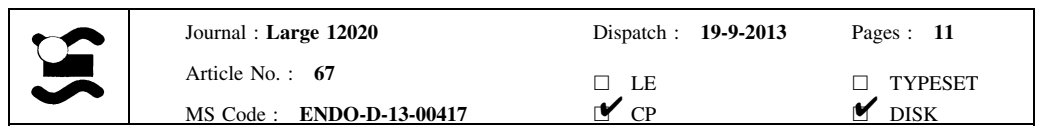


Fig. 2 The prevalence of MetS components. $B P$ blood pressure, $T G$ triglycerides, $H D L-C$ highdensity lipoprotein cholesterol, $B M I$ body mass index, $F P G$ fasting plasma glucose

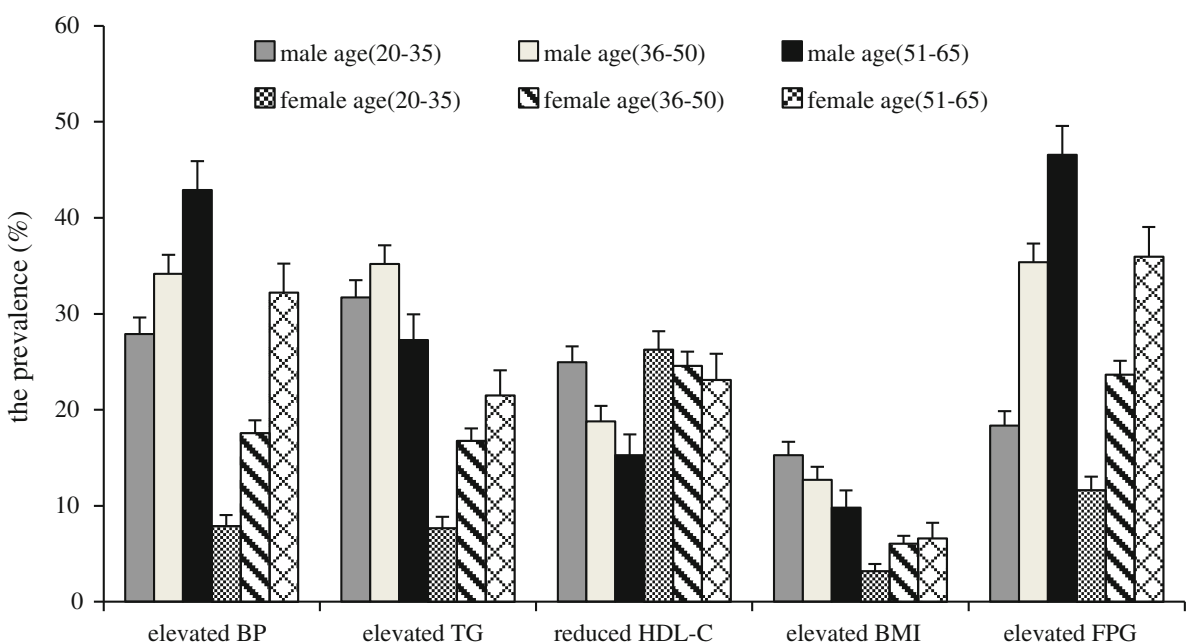

subjects, the prevalence of elevated BP, elevated TG, elevated BMI, and elevated FPG increased with age, whereas the descending trend was presented in the prevalence of reduced HDL-C. Male subjects had higher prevalence of elevated BP, elevated TG, elevated BMI, and elevated FPG levels than female subjects, whereas female subjects had a higher prevalence of reduced HDL-C level for all age groups than male subjects.

Basic characteristics and hematological parameters of subjects by age and gender

The basic characteristics and hematological parameters of 20-35-year-old subjects are displayed in Table 2. Besides the five MetS components, significant difference was found in ALT, AST, UA, WBC, lymphocytes, neutrophils, HGB, LDL-C levels, and the prevalence of taking anti-dyslipidemic drugs between MetS and non-MetS group for males. As for female subjects, significant difference was observed between the MetS group and non-MetS group for BMI, HDL-C, TG, FPG, SBP, ALT, AST, and LDL-C levels.

The basic characteristics and hematological parameters for 36-50-year-old subjects are presented in Table 3. Significant difference was found in BMI, HDL-C, TG, FPG, ALT, AST, UA, RBC, WBC, lymphocyte, neutrophil, HGB, LDL-C levels, and the prevalence of taking anti-dyslipidemic drugs between MetS and non-MetS group for males. While for females, significant difference was found in BMI, HDL-C, TG, FPG, SBP, DBP, ALT, UA, RBC, WBC, lymphocyte, neutrophil, PLT, MPV, HGB, and LDL-C levels between MetS and non-MetS group.

The basic characteristics and hematological parameters for 51-65-year-old subjects are shown in Table 4. Significant difference was found in BMI, HDL-C, TG, UA, and LDL-C levels between MetS and non-MetS group for males. As for females, significant difference was found in
BMI, HDL-C, TG, FPG, UA, HGB, and LDL-C levels between MetS and non-MetS group.

Associated risk factors for MetS by age and gender

Logistic regression analysis was used to determine associations between blood parameters and incidence of MetS after adjusted for the presence of anti-hypertensive, antidyslipidemic, and anti-diabetic medication use (Table 5; Fig. 3).

For 20-35-year-old subjects, hematological parameters positively associated with MetS were ALT (OR 2.080, $95 \%$ CI 1.371-3.159), UA (OR 2.135, $95 \%$ CI 1.294-3.614), and WBC (OR 1.482, $95 \%$ CI 1.169-2.974) for males. While neutrophils (OR 1.059, $95 \%$ CI 1.023-1.453), WBC (OR 1.398, $95 \%$ CI 1.145-3.011), and UA (OR 1.523, $95 \%$ CI 1.040-3.147) were positively associated with MetS for females.

As for 36-50-year-old subjects, ALT (OR 2.421, $95 \%$ CI 1.335-3.412), WBC (OR 2.012, $95 \%$ CI 1.290-4.010), and HGB (OR 1.045, $95 \%$ CI 1.018-2.020) were positively associated with MetS for males. While WBC (OR 3.400, $95 \%$ CI 1.818-4.528), and PLT (OR 2.616, 95 \% CI 1.432-3.033) were positively associated with MetS for females.

Hematological parameters associated with MetS for 51-65-year-old male subjects were ALT (OR 4.267, $95 \%$ CI 1.161-6.781) and LDL-C (OR 3.078, $95 \%$ CI 2.468-5.131). However, UA (OR 1.025, $95 \%$ CI 1.011-1.321), HGB (OR 1.256, $95 \%$ CI 1.145-3.105), and LDL-C (OR 2.140, $95 \%$ CI 1.524-4.359) were positively associated with MetS for 51-65-year-old females.

\section{Discussion}

The study investigated the cumulative incidence of MetS and systematically evaluated the prospective associations

\begin{tabular}{|l|lll|}
\hline & Journal : Large 12020 & Dispatch : 19-9-2013 & Pages : $\mathbf{1 1}$ \\
Article No. : $\mathbf{6 7}$ & $\square_{\mathrm{CP}}^{\mathrm{LE}}$ & $\square_{\mathrm{CISK}}^{\mathrm{TYPESET}}$ \\
\hline
\end{tabular}


Table 2 Basic characteristics and hematological parameters of 20-35-year-old subjects

\begin{tabular}{|c|c|c|c|c|c|c|}
\hline \multirow[t]{2}{*}{ Variables } & \multicolumn{3}{|l|}{ Male $(N=681)$} & \multicolumn{3}{|l|}{ Female $(N=533)$} \\
\hline & MetS & Non-MetS & $P$ value & MetS & Non-MetS & $P$ value \\
\hline$N$ & 94 & 587 & - & 22 & 511 & - \\
\hline BMI $\left(\mathrm{kg} / \mathrm{m}^{2}\right)$ & $26.56 \pm 2.84$ & $23.76 \pm 3.13$ & $<0.0001^{\mathrm{a}}$ & $24.99 \pm 3.22$ & $20.87 \pm 2.64$ & $<0.0001^{\circ}$ \\
\hline HDL-C (mmol/L) & $1.17 \pm 0.23$ & $1.32 \pm 0.27$ & $<0.0001^{\mathrm{a}}$ & $1.32(1.16-1.47)$ & $1.60(1.40-1.82)$ & $<0.0001^{\mathrm{t}}$ \\
\hline TG $(\mathrm{mmol} / \mathrm{L})$ & $1.49(1.13-2.32)$ & $1.04(0.72-1.59)$ & $<0.0001^{\mathrm{b}}$ & $1.01(0.55-1.59)$ & $0.67(0.49-0.91)$ & $0.0065^{\mathrm{t}}$ \\
\hline FPG (mmol/L) & $5.30(4.97-5.49)$ & $5.06(4.80-5.33)$ & $0.0003^{\mathrm{b}}$ & $5.41(4.85-5.49)$ & $4.99(4.74-5.24)$ & $0.0002^{\mathrm{h}}$ \\
\hline $\mathrm{SBP}(\mathrm{mmHg})$ & $120(110-130)$ & $115(110-120)$ & $0.0007^{\mathrm{b}}$ & $110(100-120)$ & $100(100-110)$ & $0.0116^{\mathrm{t}}$ \\
\hline DBP (mmHg) & $80(70-90)$ & $75(70-80)$ & $<0.0001^{\mathrm{b}}$ & $70(70-80)$ & $70(60-75)$ & $0.1419^{\mathrm{h}}$ \\
\hline $\operatorname{ALT}(\mathrm{U} / \mathrm{L})$ & $29.00(22.00-49.00)$ & $22.00(17.00-33.00)$ & $<0.0001^{\mathrm{b}}$ & $16.00(15.00-20.00)$ & $14.00(11.00-17.00)$ & $0.0101^{\mathrm{t}}$ \\
\hline AST (U/L) & $31.00(27.00-38.00)$ & $28.00(25.00-33.00)$ & $0.0014^{\mathrm{b}}$ & $29.00(24.00-32.00)$ & $26.00(23.00-29.00)$ & $0.0387^{\mathrm{t}}$ \\
\hline $\mathrm{UA}(\mu \mathrm{mol} / \mathrm{L})$ & $\begin{array}{l}390.00 \\
\quad(353.00-421.00)\end{array}$ & $\begin{array}{l}355.00 \\
\quad(320.00-396.00)\end{array}$ & $<0.0001^{\mathrm{b}}$ & $\begin{array}{l}252.00 \\
\quad(236.00-312.00)\end{array}$ & $\begin{array}{l}254.00 \\
\quad(220.00-282.00)\end{array}$ & $0.4441^{\mathrm{t}}$ \\
\hline $\mathrm{RBC}\left(\times 10^{12} / \mathrm{L}\right)$ & $5.22 \pm 0.30$ & $5.15 \pm 0.34$ & $0.0626^{\mathrm{a}}$ & $4.45 \pm 0.32$ & $4.44 \pm 0.29$ & $0.5051^{\circ}$ \\
\hline WBC $\left(\times 10^{9} / \mathrm{L}\right)$ & $6.90(5.87-7.90)$ & $6.20(5.40-7.20)$ & $0.0006^{\mathrm{b}}$ & $6.30(4.92-7.50)$ & $5.80(5.02-6.70)$ & $0.1887^{\mathrm{t}}$ \\
\hline $\begin{array}{l}\text { Lymphocyte }\left(\times 10^{9} /\right. \\
\text { L) }\end{array}$ & $2.45 \pm 0.64$ & $2.26 \pm 0.58$ & $0.0054^{\mathrm{a}}$ & $2.06 \pm 0.39$ & $2.11 \pm 0.52$ & $0.6173^{\circ}$ \\
\hline Neutrophil $\left(\times 10^{9} / \mathrm{L}\right)$ & $3.90(3.40-4.60)$ & $3.47(2.87-4.20)$ & $0.0012^{\mathrm{b}}$ & $3.95(2.80-4.70)$ & $3.39(2.78-4.00)$ & $0.0516^{\mathrm{t}}$ \\
\hline $\mathrm{MCH}(\mathrm{pg})$ & $29.80(29.30-30.80)$ & $30.10(29.20-30.80)$ & $0.5710^{\mathrm{b}}$ & $29.75(28.40-31.60)$ & $29.80(28.90-30.70)$ & $0.7727^{\mathrm{L}}$ \\
\hline PLT $\left(\times 10^{9} / \mathrm{L}\right)$ & $228.85 \pm 50.99$ & $220.37 \pm 47.48$ & $0.1140^{\mathrm{a}}$ & $248.60 \pm 54.79$ & $227.36 \pm 49.89$ & $0.0522^{\circ}$ \\
\hline MPV (fl) & $9.88 \pm 0.91$ & $9.80 \pm 1.00$ & $0.3984^{\mathrm{a}}$ & $9.75 \pm 0.85$ & $9.81 \pm 1.01$ & $0.7700^{\circ}$ \\
\hline PDW (\%) & $11.60(10.90-12.90)$ & $11.70(10.70-12.90)$ & $0.4810^{\mathrm{b}}$ & $11.65(11.10-13.00)$ & $11.80(10.80-12.90)$ & $0.7978^{\mathrm{b}}$ \\
\hline HGB $(\mathrm{g} / \mathrm{L})$ & $156.00 \pm 7.60$ & $154.15 \pm 8.99$ & $0.0348^{\mathrm{a}}$ & $\begin{array}{l}134.50 \\
(132.00-138.00)\end{array}$ & $\begin{array}{l}131.00 \\
\quad(126.00-137.00)\end{array}$ & $0.1098^{\mathrm{t}}$ \\
\hline LDL-C (mmol/L) & $3.12 \pm 0.63$ & $2.85 \pm 0.70$ & $0.0006^{\mathrm{a}}$ & $2.91(2.71-3.10)$ & $2.46(2.12-2.88)$ & $0.0002^{\mathrm{b}}$ \\
\hline \multicolumn{7}{|l|}{$\begin{array}{l}\text { Anti-hypertensive } \\
\text { drugs }\end{array}$} \\
\hline$n(\%)$ & $2(2.13)$ & $3(0.51)$ & $0.1426^{\mathrm{d}}$ & $1(4.55)$ & $5(0.98)$ & $0.2244^{\circ}$ \\
\hline \multicolumn{7}{|l|}{$\begin{array}{l}\text { Anti-dyslipidemic } \\
\text { drugs }\end{array}$} \\
\hline$n(\%)$ & $11(11.70)$ & $30(5.11)$ & $0.0126^{\mathrm{c}}$ & $1(4.55)$ & $1(0.98)$ & $0.0809^{\circ}$ \\
\hline \multicolumn{7}{|l|}{ Anti-diabetic drugs } \\
\hline$n(\%)$ & $5(5.32)$ & $26(4.43)$ & $0.6026^{\mathrm{d}}$ & $2(9.09)$ & $14(2.74)$ & $0.1376^{\mathrm{C}}$ \\
\hline
\end{tabular}

$S B P$ systolic blood pressure, $D B P$ diastolic blood pressure, $A L T$ alanine aminotransferase, $A S T$ aspartate aminotransferase, $U A$ uric acid, $R B C$ red blood cell count, $W B C$ white blood cell count, $M C H$ mean corpuscular hemoglobin, PLT platelet count, $M P V$ mean platelet volume, $P D W$ platelet distribution width, $H G B$ hemoglobin, $L D L-C$ low-density lipoprotein cholesterol

a The result of student's $t$ test

b The result of Wilcoxon rank sum test

c The result of $\chi^{2}$ test

d The result of Fisher's exact test

between several common hematological parameters and MetS by age and gender in a Beijing adult population.

The overall prevalence of MetS ranges from 6 to $38 \%$ of the general population in the United States, Europe and Asia, including Korea [21-23]. A cohort study conducted in a Taiwanese health-screening population aged 35-74 years showed that the 5-year cumulative incidence of MetS was 11.37, $14.95 \%$ for males and $9.89 \%$ for females [24]. In our study, the 5-year cumulative incidence of MetS among all subjects was $10.82 \%$, with $14.22 \%$ of males and $7.59 \%$ of females having MetS after 5-year follow-up.

Previous cross-sectional studies found that sex and age were associated with prevalence of MetS [25, 26]. It is well established that the prevalence of MetS rises from young to old ages [27, 28]. Compared with males, females had a significantly higher prevalence of central obesity and reduced HDL-C, whereas males had a significantly higher

\begin{tabular}{|l|lll|}
\hline Journal : Large 12020 & Dispatch : 19-9-2013 & Pages : $\mathbf{1 1}$ \\
& Article No. : $\mathbf{6 7}$ & $\square$ LE & $\square$ TYPESET \\
MS Code : ENDO-D-13-00417 & $\square_{\mathrm{CP}}$ & $\checkmark$ DISK \\
\hline
\end{tabular}


Table 3 Basic characteristics and hematological parameters of 36-50-year-old subjects

\begin{tabular}{|c|c|c|c|c|c|c|}
\hline \multirow[t]{2}{*}{ Variables } & \multicolumn{3}{|l|}{ Male $(N=591)$} & \multicolumn{3}{|l|}{ Female $(N=858)$} \\
\hline & MetS & Non-MetS & $P$ value & MetS & Non-MetS & $P$ value \\
\hline$N$ & 91 & 500 & - & 68 & 790 & - \\
\hline BMI $\left(\mathrm{kg} / \mathrm{m}^{2}\right)$ & $26.44 \pm 2.16$ & $24.18 \pm 2.54$ & $<0.0001^{\mathrm{a}}$ & $25.64 \pm 3.07$ & $22.57 \pm 2.62$ & $<0.0001^{\mathrm{a}}$ \\
\hline HDL-C (mmol/L) & $1.14(1.06-1.33)$ & $1.32(1.15-1.52)$ & $<0.0001^{\mathrm{b}}$ & $1.36 \pm 0.23$ & $1.66 \pm 0.34$ & $0.0257^{\mathrm{a}}$ \\
\hline $\mathrm{TG}(\mathrm{mmol} / \mathrm{L})$ & $1.83(1.41-2.83)$ & $1.20(0.87-1.73)$ & $<0.0001^{\mathrm{b}}$ & $1.23(1.02-1.65)$ & $0.86(0.64-1.20)$ & $<0.0001^{\mathrm{b}}$ \\
\hline FPG (mmol/L) & $5.53(5.26-5.90)$ & $5.32(5.03-5.61)$ & $0.0061^{\mathrm{b}}$ & $5.39(5.11-5.78)$ & $5.18(4.90-5.47)$ & $<0.0001^{\mathrm{b}}$ \\
\hline $\mathrm{SBP}(\mathrm{mmHg})$ & $120(110-125)$ & $113(105-120)$ & $0.1598^{b}$ & $120(108-125)$ & $110(100-120)$ & $<0.0001^{\mathrm{b}}$ \\
\hline DBP (mmHg) & $80(70-85)$ & $80(70-85)$ & $0.2980^{\mathrm{b}}$ & $80(70-80)$ & $70(70-80)$ & $<0.0001^{\mathrm{b}}$ \\
\hline ALT (U/L) & $29.00(22.00-39.00)$ & $22.00(18.00-31.00)$ & $<0.0001^{\mathrm{b}}$ & $17.00(14.00-22.00)$ & $15.00(12.00-20.00)$ & $0.0117^{\mathrm{b}}$ \\
\hline AST (U/L) & $33.00(29.00-39.00)$ & $30.00(26.00-34.00)$ & $0.0015^{\mathrm{b}}$ & $28.00(25.00-31.00)$ & $27.00(24.00-30.00)$ & $0.2298^{\mathrm{b}}$ \\
\hline $\mathrm{UA}(\mu \mathrm{mol} / \mathrm{L})$ & $\begin{array}{l}355.00 \\
\quad(324.00-399.00)\end{array}$ & $\begin{array}{l}338.00 \\
\quad(301.00-377.00)\end{array}$ & $0.0007^{\mathrm{b}}$ & $\begin{array}{l}275.50 \\
(243.50-305.50)\end{array}$ & $\begin{array}{l}244.00 \\
\quad(213.00-280.00)\end{array}$ & $0.0002^{\mathrm{b}}$ \\
\hline $\mathrm{RBC}\left(\times 10^{12} / \mathrm{L}\right)$ & $5.14 \pm 0.31$ & $5.03 \pm 0.34$ & $0.0026^{\mathrm{a}}$ & $4.56 \pm 0.28$ & $4.39 \pm 0.32$ & $<0.0001^{\mathrm{a}}$ \\
\hline WBC $\left(\times 10^{9} / \mathrm{L}\right)$ & $6.80(5.80-8.03)$ & $6.10(5.25-7.30)$ & $<0.0001^{\mathrm{b}}$ & $6.45(5.70-7.70)$ & $5.70(4.90-6.60)$ & $<0.0001^{\mathrm{b}}$ \\
\hline $\begin{array}{l}\text { Lymphocyte }\left(\times 10^{9} /\right. \\
\text { L) }\end{array}$ & $2.30(2.00-2.70)$ & $2.10(1.70-2.50)$ & $0.0043^{\mathrm{b}}$ & $2.17(1.90-2.50)$ & $1.90(1.60-2.20)$ & $<0.0001^{\mathrm{b}}$ \\
\hline Neutrophil $\left(\times 10^{9} / \mathrm{L}\right)$ & $4.10(3.40-5.00)$ & $3.50(2.90-4.30)$ & $<0.0001^{\mathrm{b}}$ & $3.90(3.45-4.62)$ & $3.40(2.76-4.10)$ & $<0.0001^{\mathrm{b}}$ \\
\hline $\mathrm{MCH}(\mathrm{pg})$ & $30.53 \pm 1.45$ & $30.42 \pm 1.79$ & $0.5371^{\mathrm{a}}$ & $29.60(28.65-30.90)$ & $30.10(29.10-30.90)$ & $0.2029^{\mathrm{b}}$ \\
\hline $\operatorname{PLT}\left(\times 10^{9} / \mathrm{L}\right)$ & $214.10 \pm 46.17$ & $213.06 \pm 46.11$ & $0.8438^{\mathrm{a}}$ & $262.30 \pm 60.03$ & $228.68 \pm 50.00$ & $<0.0001^{\mathrm{a}}$ \\
\hline MPV (fl) & $9.40(8.90-10.10)$ & $9.40(8.70-10.10)$ & $0.4320^{\mathrm{b}}$ & $9.20(8.65-10.00)$ & $9.50(8.90-10.20)$ & $0.0265^{\mathrm{b}}$ \\
\hline PDW (\%) & $11.50(10.80-12.80)$ & $11.40(10.30-12.70)$ & $0.3026^{\mathrm{b}}$ & $11.15(10.40-12.00)$ & $11.60(10.60-12.80)$ & $0.0501^{\mathrm{b}}$ \\
\hline HGB $(\mathrm{g} / \mathrm{L})$ & $156.80 \pm 9.43$ & $152.47 \pm 8.76$ & $<0.0001^{\mathrm{a}}$ & $\begin{array}{l}136.00 \\
(130.00-139.00)\end{array}$ & $\begin{array}{l}131.00 \\
(125.00-137.00)\end{array}$ & $0.0009^{b}$ \\
\hline LDL-C (mmol/L) & $3.29 \pm 0.78$ & $3.10 \pm 0.72$ & $0.0249^{\mathrm{a}}$ & $3.07(2.63-3.32)$ & $2.77(2.42-3.31)$ & $0.0119^{\mathrm{b}}$ \\
\hline \multicolumn{7}{|l|}{$\begin{array}{l}\text { Anti-hypertensive } \\
\text { drugs }\end{array}$} \\
\hline$n(\%)$ & $15(16.48)$ & $55(11.00)$ & $0.1365^{\mathrm{c}}$ & $7(10.29)$ & $43(5.44)$ & $0.1059^{\mathrm{d}}$ \\
\hline \multicolumn{7}{|l|}{$\begin{array}{l}\text { Anti-dyslipidemic } \\
\text { drugs }\end{array}$} \\
\hline$n(\%)$ & $19(20.88)$ & $63(12.60)$ & $0.0356^{\mathrm{c}}$ & $1(1.47)$ & $10(1.27)$ & $0.5990^{\mathrm{d}}$ \\
\hline \multicolumn{7}{|l|}{ Anti-diabetic drugs } \\
\hline$n(\%)$ & $17(18.68)$ & $57(11.40)$ & $0.0536^{\mathrm{c}}$ & $6(8.82)$ & $32(4.05)$ & $0.1125^{\mathrm{d}}$ \\
\hline
\end{tabular}

$S B P$ systolic blood pressure, $D B P$ diastolic blood pressure, $A L T$ alanine aminotransferase, $A S T$ aspartate aminotransferase, $U A$ uric acid, $R B C$ red blood cell count, $W B C$ white blood cell count, $M C H$ mean corpuscular hemoglobin, PLT platelet count, $M P V$ mean platelet volume, $P D W$ platelet distribution width, $H G B$ hemoglobin, $L D L-C$ low-density lipoprotein cholesterol

a The result of student's $t$ test

b The result of Wilcoxon rank sum test

c The result of $\chi^{2}$ test

d The result of Fisher's exact test

prevalence of raised BP compared with females [29]. Our results are roughly consistent with these observations. In this study, the incidence of MetS increased with age for females, while the same trend was not found for males. The prevalence of elevated BP and FPG increased with age for male subjects, and the prevalence of elevated $\mathrm{BP}$, TG, BMI, and FPG increased with age for female subjects, whereas the descending trend was presented in the prevalence of reduced HDL-C for female subjects. Information from our study suggests that males are more prone to be affected by MetS compared to females for young and middle-age people, while females are at higher risk for MetS for elderly people. The incidence of MetS and prevalence of its components in this population underlines the need to screen for associated hematological factors for MetS.

Several reports have demonstrated that altered hematological status in patients is a high risk factor for MetS.

\begin{tabular}{|l|lll|}
\hline & Journal : Large 12020 & Dispatch : 19-9-2013 & Pages : $\mathbf{1 1}$ \\
Article No. : $\mathbf{6 7}$ & $\square_{\mathrm{CP}}^{\mathrm{LE}}$ & $\square_{\mathrm{CISK}}^{\mathrm{TYPESET}}$ \\
\hline
\end{tabular}


Table 4 Basic characteristics and hematological parameters of 51-65-year-old subjects

\begin{tabular}{|c|c|c|c|c|c|c|}
\hline \multirow[t]{2}{*}{ Variables } & \multicolumn{3}{|l|}{ Male $(N=275)$} & \multicolumn{3}{|l|}{ Female $(N=242)$} \\
\hline & MetS & Non-MetS & $P$ value & MetS & Non-MetS & $P$ value \\
\hline$N$ & 35 & 240 & - & 34 & 208 & - \\
\hline BMI $\left(\mathrm{kg} / \mathrm{m}^{2}\right)$ & $26.36 \pm 2.63$ & $24.50 \pm 2.85$ & $0.0009^{\mathrm{a}}$ & $25.97 \pm 2.95$ & $23.32 \pm 2.59$ & $<0.0001^{\circ}$ \\
\hline HDL-C (mmol/L) & $1.18(1.09-1.29)$ & $1.30(1.13-1.51)$ & $0.0070^{\mathrm{b}}$ & $1.42 \pm 0.30$ & $1.69 \pm 0.34$ & $<0.0001^{\circ}$ \\
\hline $\mathrm{TG}(\mathrm{mmol} / \mathrm{L})$ & $1.89(1.28-3.43)$ & $1.24(0.87-1.65)$ & $<0.0001^{\mathrm{b}}$ & $1.58(1.30-1.85)$ & $1.10(0.78-1.55)$ & $<0.0001^{\mathrm{l}}$ \\
\hline FPG (mmol/L) & $5.47(4.97-6.04)$ & $5.44(5.11-5.83)$ & $0.8788^{\mathrm{b}}$ & $5.57(5.22-6.06)$ & $5.37(5.00-5.77)$ & $0.0428^{\mathrm{t}}$ \\
\hline $\mathrm{SBP}(\mathrm{mmHg})$ & $120(110-125)$ & $120(105-125)$ & $0.2890^{\mathrm{b}}$ & $120(110-130)$ & $120(105-125)$ & $0.2501^{\mathrm{b}}$ \\
\hline $\mathrm{DBP}(\mathrm{mmHg})$ & $75(70-85)$ & $80(70-85)$ & $1.0000^{\mathrm{b}}$ & $80(70-85)$ & $80(70-80)$ & $0.4339^{\mathrm{l}}$ \\
\hline ALT (U/L) & $21.50(18.00-31.00)$ & $20.00(17.00-28.00)$ & $0.2591^{\mathrm{b}}$ & $20.00(15.00-29.00)$ & $18.00(14.00-23.00)$ & $0.1268^{\mathrm{b}}$ \\
\hline AST (U/L) & $27.50(25.00-33.00)$ & $29.00(26.00-35.00)$ & $0.2247^{\mathrm{b}}$ & $28.50(25.00-32.00)$ & $29.00(26.00-33.00)$ & $0.6593^{\mathrm{t}}$ \\
\hline $\mathrm{UA}(\mu \mathrm{mol} / \mathrm{L})$ & $364.30 \pm 65.78$ & $340.21 \pm 66.90$ & $0.0471^{\mathrm{a}}$ & $295.20 \pm 51.90$ & $270.30 \pm 58.50$ & $0.0204^{\circ}$ \\
\hline $\mathrm{RBC}\left(\times 10^{12} / \mathrm{L}\right)$ & $5.00(4.79-5.21)$ & $4.88(4.65-5.12)$ & $0.1854^{\mathrm{b}}$ & $4.41(4.34-4.76)$ & $4.42(4.21-4.61)$ & $0.1263^{\mathrm{t}}$ \\
\hline $\mathrm{WBC}\left(\times 10^{9} / \mathrm{L}\right)$ & $7.13(5.90-7.72)$ & $6.30(5.30-7.40)$ & $0.0830^{\mathrm{b}}$ & $6.09(5.00-6.71)$ & $5.53(4.80-6.68)$ & $0.2791^{\mathrm{b}}$ \\
\hline $\begin{array}{l}\text { Lymphocyte }\left(\times 10^{9} /\right. \\
\text { L) }\end{array}$ & $2.40(1.73-2.70)$ & $2.10(1.70-2.60)$ & $0.2712^{\mathrm{b}}$ & $2.15(1.90-2.60)$ & $2.00(1.69-2.40)$ & $0.1411^{\mathrm{b}}$ \\
\hline Neutrophil $\left(\times 10^{9} / \mathrm{L}\right)$ & $4.20(3.20-4.80)$ & $3.60(3.00-4.56)$ & $0.1119^{\mathrm{b}}$ & $3.15(2.63-4.01)$ & $3.20(2.50-3.90)$ & $0.5432^{\mathrm{h}}$ \\
\hline $\mathrm{MCH}(\mathrm{pg})$ & $31.00(29.80-31.40)$ & $30.80(29.90-31.80)$ & $0.6847^{\mathrm{b}}$ & $30.05(29.30-31.10)$ & $29.95(29.10-30.90)$ & $0.4624^{\mathrm{b}}$ \\
\hline $\operatorname{PLT}\left(\times 10^{9} / \mathrm{L}\right)$ & $\begin{array}{l}196.00 \\
(175.00-239.00)\end{array}$ & $\begin{array}{l}205.00 \\
\quad(180.00-242.00)\end{array}$ & $0.9187^{b}$ & $\begin{array}{l}214.00 \\
\quad(185.00-249.00)\end{array}$ & $\begin{array}{l}218.00 \\
\quad(189.00-256.00)\end{array}$ & $0.7302^{\mathrm{h}}$ \\
\hline MPV (fl) & $9.30(8.80-9.80)$ & $9.30(8.60-9.90)$ & $0.8346^{\mathrm{b}}$ & $9.55(8.80-10.30)$ & $9.40(8.90-10.10)$ & $0.7320^{\mathrm{t}}$ \\
\hline PDW (\%) & $11.40(10.40-12.40)$ & $11.30(10.30-12.30)$ & $0.7369^{\mathrm{b}}$ & $11.25(10.70-12.40)$ & $11.55(10.60-12.40)$ & $0.9842^{\mathrm{L}}$ \\
\hline HGB $(\mathrm{g} / \mathrm{L})$ & $152.60 \pm 8.94$ & $151.30 \pm 8.87$ & $0.4173^{\mathrm{a}}$ & $135.90 \pm 8.65$ & $131.39 \pm 9.25$ & $0.0091^{\circ}$ \\
\hline LDL-C (mmol/L) & $3.46 \pm 0.86$ & $3.16 \pm 0.70$ & $0.0266^{\mathrm{a}}$ & $3.67(3.38-4.31)$ & $3.43(2.86-3.90)$ & $0.0227^{\mathrm{t}}$ \\
\hline \multicolumn{7}{|l|}{$\begin{array}{l}\text { Anti-hypertensive } \\
\text { drugs }\end{array}$} \\
\hline$n(\%)$ & $9(25.71)$ & $42(17.50)$ & $0.2428^{c}$ & $10(29.41)$ & $37(17.79)$ & $0.1576^{\mathrm{C}}$ \\
\hline \multicolumn{7}{|l|}{$\begin{array}{l}\text { Anti-dyslipidemic } \\
\text { drugs }\end{array}$} \\
\hline$n(\%)$ & $4(11.43)$ & $33(13.75)$ & $1.0000^{\mathrm{d}}$ & $3(8.82)$ & $12(5.77)$ & $0.4496^{\circ}$ \\
\hline \multicolumn{7}{|l|}{ Anti-diabetic drugs } \\
\hline$n(\%)$ & $6(17.14)$ & $31(12.92)$ & $0.4388^{\mathrm{d}}$ & $6(17.65)$ & $22(10.58)$ & $0.2477^{\circ}$ \\
\hline
\end{tabular}

$S B P$ systolic blood pressure, $D B P$ diastolic blood pressure, $A L T$ alanine aminotransferase, $A S T$ aspartate aminotransferase, $U A$ uric acid, $R B C$ red blood cell count, $W B C$ white blood cell count, $M C H$ mean corpuscular hemoglobin, $P L T$ platelet count, $M P V$ mean platelet volume, $P D W$ platelet distribution width, $H G B$ hemoglobin, $L D L-C$ low-density lipoprotein cholesterol

a The result of student's $t$ test

b The result of Wilcoxon rank sum test

c The result of $\chi^{2}$ test

d The result of Fisher's exact test

Elevated ALT was found to be predictive of MetS among adolescents and young adults in mainland China [30]. The prevalence of MetS increases with the increase in blood levels of ALT even through the normal range of ALT in Japanese men and women [31]. Our results showed that ALT was significantly associated with the incidence of MetS only for males. The result indicated that the association between ALT and MetS was gender-specific.

There are significant associations among UA, CVD, and MetS [32, 33], partly explained by the activation of the renin-angiotensin system by obesity [34] or vascular dysfunction including inflammation. In $\equiv$ ition, nutritional factors are speculated to affect the occurfence of MetS and also of UA. Significant association between UA and MetS was found in 20-35-year-old males and females, and 51-65-year-old females.

Jesri et al. [35] reported that subjects with MetS had AQ1 96 higher PLT and $\equiv \mathrm{BC}$ counts than controls, and these two 297 parameters linearry increased as the number of MetS 298 components increased. WBC was associated with MetS 299

\begin{tabular}{|l|lll|} 
Journal : Large 12020 & Dispatch : 19-9-2013 & Pages : $\mathbf{1 1}$ \\
Article No. : 67 & $\square$ LE & $\square$ TYPESET \\
MS Code : ENDO-D-13-00417 & $\sim_{\mathrm{CP}}$ & $\boldsymbol{\sim}$ DISK \\
\hline
\end{tabular}


Table 5 Associated risk factors of MetS by gender and age

\begin{tabular}{|c|c|c|c|c|c|c|c|c|}
\hline \multirow[t]{2}{*}{ Gender } & \multirow[t]{2}{*}{ Age at inclusion } & \multirow[t]{2}{*}{ Parameter } & \multirow[t]{2}{*}{ Estimate } & \multirow[t]{2}{*}{ Standard error } & \multirow[t]{2}{*}{$P$ value } & \multirow[t]{2}{*}{ OR } & \multicolumn{2}{|c|}{$95 \% \mathrm{CI}$ for OR } \\
\hline & & & & & & & Lower & Upper \\
\hline \multirow[t]{8}{*}{ Male } & \multirow[t]{3}{*}{$20-35$} & ALT & 0.733 & 0.213 & 0.0006 & 2.080 & 1.371 & 3.159 \\
\hline & & UA & 0.758 & 0.326 & 0.0004 & 2.135 & 1.294 & 3.614 \\
\hline & & WBC & 0.393 & 0.154 & 0.0071 & 1.482 & 1.169 & 2.974 \\
\hline & \multirow[t]{3}{*}{$36-50$} & ALT & 0.884 & 0.306 & 0.0039 & 2.421 & 1.335 & 3.412 \\
\hline & & WBC & 0.699 & 0.208 & 0.0021 & 2.012 & 1.290 & 4.010 \\
\hline & & HGB & 0.044 & 0.018 & 0.0480 & 1.045 & 1.018 & 2.020 \\
\hline & \multirow[t]{2}{*}{$51-65$} & ALT & 1.451 & 0.664 & 0.0289 & 4.267 & 1.161 & 6.781 \\
\hline & & LDL-C & 1.124 & 0.584 & 0.0176 & 3.078 & 2.468 & 5.131 \\
\hline \multirow[t]{8}{*}{ Female } & \multirow[t]{3}{*}{$20-35$} & neutrophil & 0.349 & 0.169 & 0.0356 & 1.059 & 1.023 & 1.453 \\
\hline & & WBC & 0.335 & 0.116 & 0.0124 & 1.398 & 1.145 & 3.011 \\
\hline & & UA & 0.421 & 0.211 & 0.0341 & 1.523 & 1.040 & 3.147 \\
\hline & \multirow[t]{2}{*}{$36-50$} & WBC & 1.224 & 0.642 & 0.0038 & 3.400 & 1.818 & 4.528 \\
\hline & & PLT & 0.962 & 0.697 & 0.0133 & 2.616 & 1.432 & 3.033 \\
\hline & \multirow[t]{3}{*}{$51-65$} & UA & 0.005 & 0.003 & 0.0298 & 1.025 & 1.011 & 1.321 \\
\hline & & HGB & 0.228 & 0.012 & 0.0164 & 1.256 & 1.145 & 3.105 \\
\hline & & LDL-C & 0.761 & 0.247 & 0.0231 & 2.140 & 1.524 & 4.359 \\
\hline
\end{tabular}

Model was adjusted for the presence of anti-hypertensive, anti-dyslipidemic, and anti-diabetic medication use

$A L T$ alanine aminotransferase, $U A$ uric acid, $W B C$ white blood cell count, $P L T$ platelet count, $H G B$ hemoglobin, $L D L-C$ low-density lipoprotein cholesterol

and its individual components [36]. In this study, WBC was found to be associated with $=$ etS in 20-35-year-old and 36-50-year-old groups for indicated that WBC is strongly associated with MetS for young adults. In this study, young adults have higher prevalence of elevated TG, reduced HDL-C, and obesity than elderly people.

Several studies showed that both obesity and dyslipidemia were the major precursors for development of MetS, and perivascular white adipose tissue can release proinflammatory cytokines [37, 38], such as IL-8, leading to elevated WBC, especially $\equiv$ monocytes and granulocytes. In addition, TNF- $\alpha$ is showrr to be constitutively expressed by adipose tissue, and this proinflammatory cytokines leads to elevated WBC $[39,40]$. Therefore, the total WBC

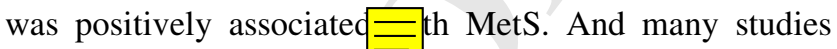
have shown the similar restrts. One study conducted on a Japanese population showed that the correlation between WC and CRP was significantly stronger in younger men than in older men [36]. And, another study displayed a correlation between $\equiv \mathrm{m} \mathrm{CRP}$ and WBC and some CVD risk factors among young adults [41]. Significant correlation of CRP with BMI and WC was discovered in adolescents and young adults in one study conducted in Asian Indians [42].

RBC counts and HGB were associated with MetS and its components in men and women [43]. Our results showed that HGB was positively associated with MetS for 36-50year-old male subjects and 51-65-year-old female subjects.

Neutrophil counts were significantly increased in MetS [44]. In this study, significant association between neutrophil counts and the incidence of MetS was found in 20-35year-old female subjects.

Park et al. [45] reported that PLT and MPV might be a surrogate ma $\equiv$ associated with clustered MetS in women. PLT with MetS components [17], and our results showed the same association between PLT and MetS for 36-50-yearold female subjects.

Subjects with MetS had elevated levels of oxidized LDL [46]. Circulating oxidized LDL seems to express the level of oxidative stress and associate with the risk factors of MetS [47]. A strongly positive association between LDL-C and MetS was found for 51-65-year-old male and female subjects. It indicates that LDL-C is strongly associated with MetS for elderly people.

Although several reports have demonstrated that there is a close relationship between RBC, MPV, and MetS [4850], no positive associations between these hematological parameters and MetS were found in our study.

There were some limitations to this study. First, hematological parameters were assessed from a single blood sample in the study, and therefore intra-individual variation cannot be taken into account. Second, information about

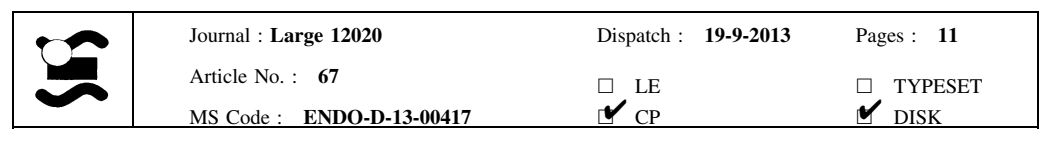




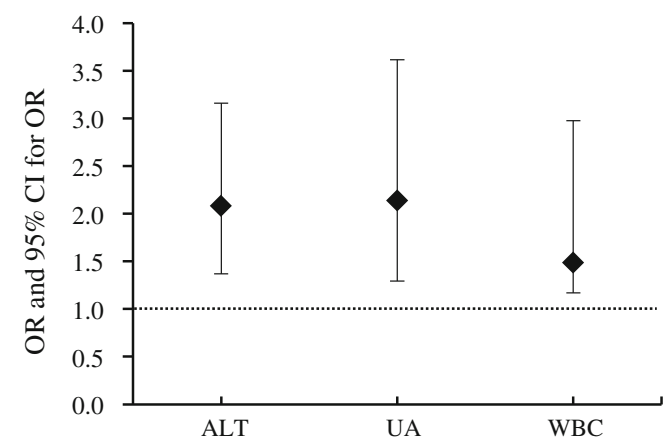

a Risk factors for 20-35-year-old male subjects

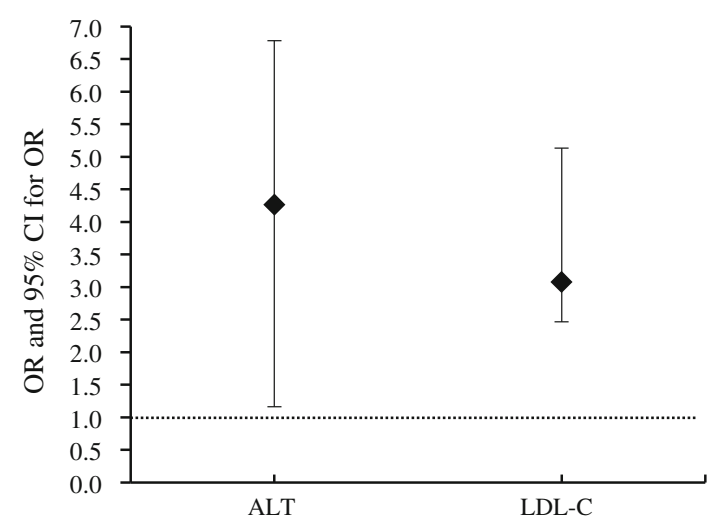

c Risk factors for 51-65-year-old male subjects

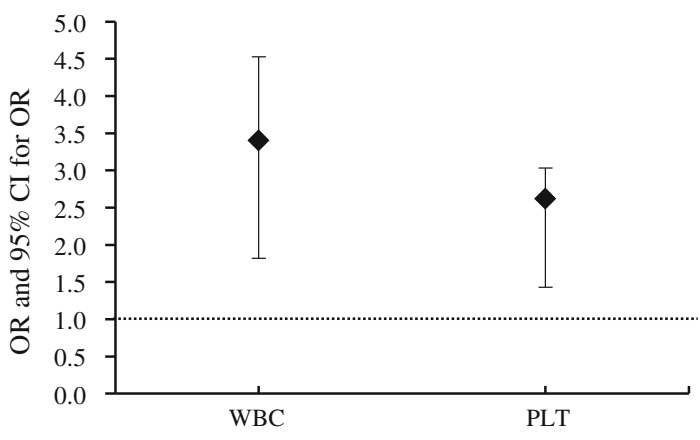

e Risk factors for 36-50-year-old female subjects

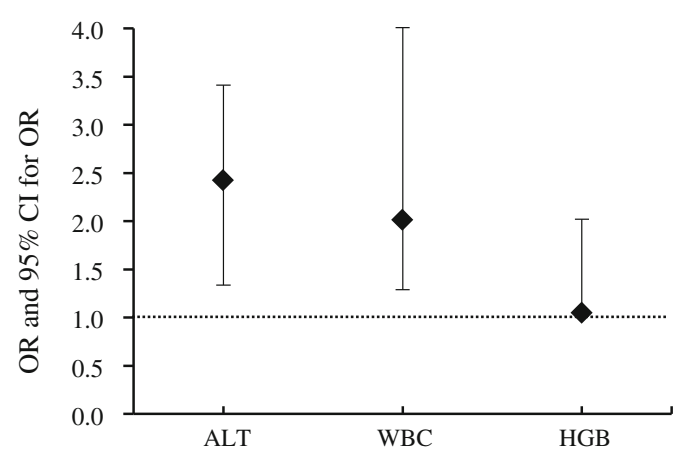

b Risk factors for 36-50-year-old male subjects

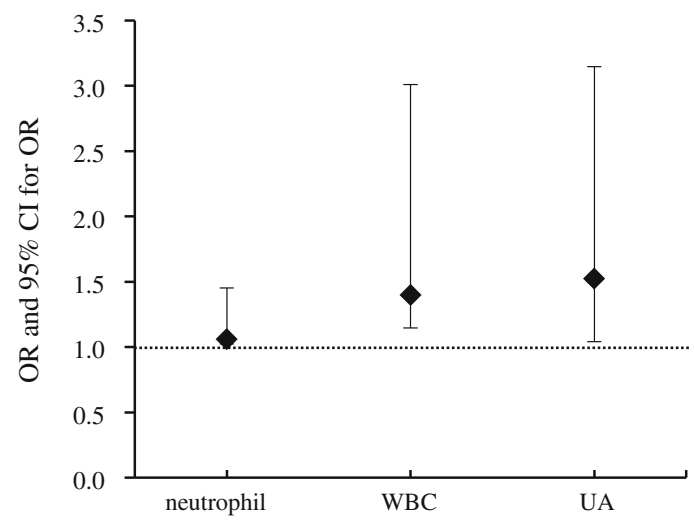

d Risk factors for 20-35-year-old female subjects

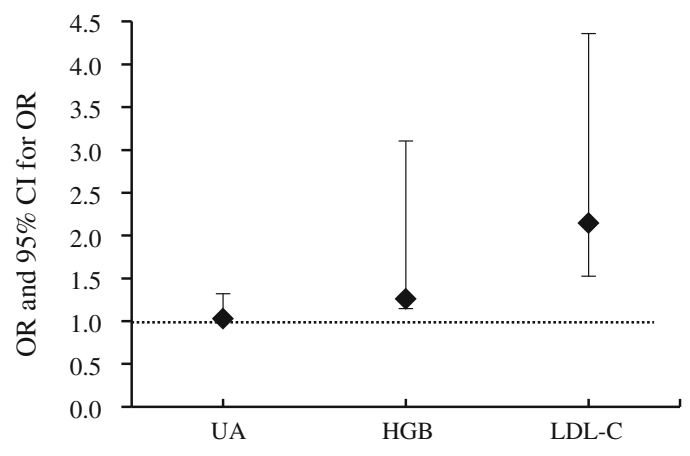

f Risk factors for 51-65-year-old female subjects

Fig. 3 OR and $95 \% \mathrm{CI}$ for risk factors of MetS. $A L T$ alanine aminotransferase, $U A$ uric acid, $W B C$ white blood cell count, $H G B$ hemoglobin, $L D L-C$ low-density lipoprotein cholesterol, $P L T$ platelet count, $O R$ odds ratio, $C I$ confidence interval

lifestyles was not available, and the multivariate model was not adjusted for these factors. But the lifestyle variables will be included in further studies. Third, as Beijing Tongren Hospital is located in the urban area of Beijing, selection bias may be that there were more people with modern life style recruited for the research. In addition, the study was based on a population attending for routine health check-up from one single hospital. Therefore, the demographics and referral source may limit the generalization of the results. And further studies using the general population would be desirable.

\section{Conclusions}

Our study sample showed that the 5-year cumulative incidence of MetS was $10.82 \%$, with $14.22 \%$ of males and $7.59 \%$ of females having MetS after 5-year follow-up. Among all the hematological parameters, WBC is positively associated with MetS for young adults, while LDL-C is positively associated with MetS for elderly people. ALT is positively associated with MetS for males only. The association between WBC, LDL-C, and MetS was agespecific. While the association between ALT and MetS was

\begin{tabular}{|l|ll|}
\hline Journal : Large 12020 & Dispatch : 19-9-2013 & Pages : 11 \\
Article No. : 67 & $\square$ LE & $\square$ TYPESET \\
MS Code : ENDO-D-13-00417 & $\boldsymbol{V}_{\mathrm{CP}}$ & $\boldsymbol{\sim}$ DISK \\
\hline
\end{tabular}


gender-specific. The study provides further evidence in support of using hematological markers for early detection of different age groups of individuals at risk of MetS.

Acknowledgments The study has been funded by the Major Project of Natural Science Fund of Beijing (Serial Number: 7131002); Key Projects in the National Science \& Technology Pillar Program in the Twelfth Five-year Plan Period of China (2011BAI08B01); the Program of Natural Science Fund of China (Serial Number: 81373099). We also would like to thank the medical personnel who participated in data collection in the physical check-up department of Beijing TongRen Hospital.

Conflict of interest The authors declare no conflict of interest.

\section{References}

1. R. Dajani, Y.S. Khader, N. Hakooz, R. Fatahalla, F. Quadan, Metabolic syndrome between two ethnic minority groups (Circassians and Chechens) and the original inhabitants of Jordan. Endocrine 43(1), 112-119 (2013)

2. L. Guize, F. Thomas, B. Pannier, K. Bean, B. Jego, A. Benetos, All-cause mortality associated with specific combinations of the metabolic syndrome according to recent definitions. Diabetes Care 30(9), 2381-2387 (2007)

3. X.H. Guo, P.H. Zhang, Z.C. Zeng, W. Wang, C. Li, Y. Shi, Z.J. Liu, Combination patterns of cardiovascular risks and sequelae at different stage of hypertension in natural hypertensive population in Beijing. Clin. Exp. Hypertens. 31(2), 142-155 (2009)

4. C.C. Chen, W.Y. Lin, C.I. Li, C.S. Liu, T.C. Li, Y.T. Chen, C.W. Yang, M.P. Chang, C.C. Lin, The association of alcohol consumption with metabolic syndrome and its individual components: the Taichung community health study. Nutr. Res. 32(1), 24-29 (2012)

5. A. Esteghamati, A. Rashidi, O. Khalilzadeh, H. Ashraf, M. Abbasi, Metabolic syndrome is independently associated with microalbuminuria in type 2 diabetes. Acta Diabetol. 47(2), 125-130 (2010)

6. J. Zhang, Y. Chen, Y. Xu, M. Li, T. Wang, B. Xu, J. Sun, M. Xu, J. Lu, Y. Bi, Low-grade albuminuria is associated with metabolic syndrome and its components in middle-aged and elderly Chinese population. PLoS ONE 8(6), e65597 (2013)

7. A.S. Cardoso, N.C. Gonzaga, C.C. Medeiros, D.F. de Carvalho, Association of uric acid levels with components of metabolic syndrome and non-alcoholic fatty liver disease in overweight or obese children and adolescents. J. Pediatr. (Rio J) 89(4), 412-418 (2013)

8. J.P. Goncalves, A. Oliveira, M. Severo, A.C. Santos, C. Lopes, Cross-sectional and longitudinal associations between serum uric acid and metabolic syndrome. Endocrine 41(3), 450-457 (2012)

9. G.P. Fadini, G. Marcuzzo, M.C. Marescotti, S.V. de Kreutzenberg, A. Avogaro, Elevated white blood cell count is associated with prevalence and development of the metabolic syndrome and its components in the general population. Acta Diabetol. 49(6), 445-451 (2012)

10. E. Oda, High-sensitivity C-reactive protein and white blood cell count equally predict development of the metabolic syndrome in a Japanese health screening population. Acta Diabetol. (2013)

11. M. Barbieri, E. Ragno, E. Benvenuti, G.A. Zito, A. Corsi, L. Ferrucci, G. Paolisso, New aspects of the insulin resistance syndrome: impact on haematological parameters. Diabetologia 44(10), 1232-1237 (2001)

12. E.S. Ford, W.H. Giles, W.H. Dietz, Prevalence of the metabolic syndrome among US adults: findings from the third National
Health and Nutrition Examination Survey. JAMA 287(3), 356-359 (2002)

13. K. Lee, J.H. Yang, Which liver enzymes are better indicators of metabolic syndrome in adolescents: the Fifth Korea National Health and Nutrition Examination Survey, 2010. Metab. Syndr. Relat. Disord. 11(4), 229-235 (2013)

14. Y. Zhang, X. Lu, J. Hong, M. Chao, W. Gu, W. Wang, G. Ning, Positive correlations of liver enzymes with metabolic syndrome including insulin resistance in newly diagnosed type 2 diabetes mellitus. Endocrine 38(2), 181-187 (2010)

15. J. Jo, J.E. Yun, H. Lee, H. Kimm, S.H. Jee, Total, direct, and indirect serum bilirubin concentrations and metabolic syndrome among the Korean population. Endocrine 39(2), 182-189 (2011)

16. R. Kawamoto, Y. Tabara, K. Kohara, T. Miki, T. Kusunoki, M. Abe, T. Katoh, Hematological parameters are associated with metabolic syndrome in Japanese community-dwelling persons. Endocrine 43(2), 334-341 (2013)

17. V. Lohsoonthorn, W. Jiamjarasrungsi, M.A. Williams, Association of hematological parameters with clustered components of metabolic syndrome among professional and office workers in Bangkok, Thailand. Diabetes Metab. Syndr. 1(3), 143-149 (2007)

18. T. Yang, C.H. Chu, P.C. Hsieh, C.H. Hsu, Y.C. Chou, S.H. Yang, C.H. Bai, S.L. You, L.C. Hwang, T.C. Chung, C.A. Sun, C-reactive protein concentration as a significant correlate for metabolic syndrome: a Chinese population-based study. Endocrine 43(2), 351-359 (2013)

19. K.G. Alberti, R.H. Eckel, S.M. Grundy, P.Z. Zimmet, J.I. Cleeman, K.A. Donato, J.C. Fruchart, W.P. James, C.M. Loria, S.C. Smith Jr, Harmonizing the metabolic syndrome: a joint interim statement of the International Diabetes Federation Task Force on Epidemiology and Prevention; National Heart, Lung, and Blood Institute; American Heart Association; World Heart Federation; International Atherosclerosis Society; and International Association for the Study of Obesity. Circulation 120(16), 1640-1645 (2009)

20. C. Chen, F.C. Lu, The guidelines for prevention and control of overweight and obesity in Chinese adults. Biomed. Environ. Sci. 17(Suppl), 1-36 (2004)

21. V. Lohsoonthorn, S. Lertmaharit, M.A. Williams, Prevalence of metabolic syndrome among professional and office workers in Bangkok, Thailand. J. Med. Assoc. Thai. 90(9), 1908-1915 (2007)

22. H.S. Park, S.W. Oh, S.I. Cho, W.H. Choi, Y.S. Kim, The metabolic syndrome and associated lifestyle factors among South Korean adults. Int. J. Epidemiol. 33(2), 328-336 (2004)

23. W. Wang, Y. Luo, Y. Liu, C. Cui, L. Wu, Y. Wang, H. Wang, P. Zhang, X. Guo, Prevalence of metabolic syndrome and optimal waist circumference cut-off points for adults in Beijing. Diabetes Res. Clin. Pract. 88(2), 209-216 (2010)

24. X. Yang, Q. Tao, F. Sun, S. Zhan, The impact of socioeconomic status on the incidence of metabolic syndrome in a Taiwanese health screening population. Int. J. Public Health 57(3), 551-559 (2012)

25. D. Hu, P. Fu, J. Xie, C.S. Chen, D. Yu, P.K. Whelton, J. He, D. $\mathrm{Gu}$, Increasing prevalence and low awareness, treatment and control of diabetes mellitus among Chinese adults: the InterASIA study. Diabetes Res. Clin. Pract. 81(2), 250-257 (2008)

26. Y. Pan, C.A. Pratt, Metabolic syndrome and its association with diet and physical activity in US adolescents. J. Am. Diet. Assoc. 108(2), 276-286; discussion 286 (2008)

27. A.J. Cameron, J.E. Shaw, P.Z. Zimmet, The metabolic syndrome: prevalence in worldwide populations. Endocrinol. Metab. Clin. N. Am. 33(2), 351-375 (2004)

28. W. Yang, K. Reynolds, D. Gu, J. Chen, J. He, A comparison of two proposed definitions for metabolic syndrome in the Chinese adult population. Am. J. Med. Sci. 334(3), 184-189 (2007)

\begin{tabular}{|c|c|c|c|c|}
\hline & Journal : Large 12020 & Dispatch : & $19-9-2013$ & Pages: $\mathbf{1 1}$ \\
\hline & $\begin{array}{l}\text { Article No. : } 67 \\
\text { MS Code : } \quad \text { ENDO-D-13-00417 }\end{array}$ & $\begin{array}{l}\square \mathrm{LE} \\
\boldsymbol{\sim}_{\mathrm{CP}} \\
\end{array}$ & & $\begin{array}{l}\square \\
\checkmark \text { TYPESET } \\
\text { DISK }\end{array}$ \\
\hline
\end{tabular}


29. X.Y. Wu, C.L. Hu, Y.H. Wan, P.Y. Su, C. Xing, X.Y. Qi, F.B. Tao, Higher waist-to-height ratio and waist circumference are predictive of metabolic syndrome and elevated serum alanine aminotransferase in adolescents and young adults in mainland China. Public Health 126(2), 135-142 (2012)

30. E. Oda, R. Kawai, K. Watanabe, V. Sukumaran, Prevalence of metabolic syndrome increases with the increase in blood levels of gamma glutamyltransferase and alanine aminotransferase in Japanese men and women. Intern. Med. 48(16), 1343-1350 (2009)

31. F. Galletti, P. Strazzullo, Involvement of the renin-angiotensin system in obesity: older and newer pathways. Nutr. Metab. Cardiovasc. Dis. 17(10), 699-704 (2007)

32. C. Li, M.C. Hsieh, S.J. Chang, Metabolic syndrome, diabetes, and hyperuricemia. Curr. Opin. Rheumatol. 25(2), 210-216 (2013)

33. R. Sarzani, F. Salvi, P. Dessi-Fulgheri, A. Rappelli, Reninangiotensin system, natriuretic peptides, obesity, metabolic syndrome, and hypertension: an integrated view in humans. J. Hypertens. 26(5), 831-843 (2008)

34. A. Jesri, E.C. Okonofua, B.M. Egan, Platelet and white blood cell counts are elevated in patients with the metabolic syndrome. J. Clin. Hypertens. (Greenwich) 7(12), 705-711; quiz 712-713 (2005)

35. K. Kotani, N. Sakane, K. Saiga, H. Mu, Y. Kurozawa, Clustered components of the metabolic syndrome and platelet counts in Japanese females. Clin. Chem. Lab. Med. 45(3), 376-379 (2007)

36. E. Oda, R. Kawai, Age- and gender-related differences in corE relations between abdominal obesity and obesity-related metabolic risk factors in Japanese. Intern. Med. 48(7), 497-502 (2009)

37. S. Hagita, M. Osaka, K. Shimokado, M. Yoshida, Adipose

- inflammation initiates recruitment of leukocytes to mouse femoral artery: role of adipo-vascular axis in chronic inflammation. PLoS ONE 6(5), e19871 (2011)

38. N. Kawanishi, H. Yano, Y. Yokogawa, K. Suzuki, Exercise training inhibits inflammation in adipose tissue via both sup- pression of macrophage infiltration and acceleration of phenotypic switching from M1 to M2 macrophages in high-fat-dietinduced obese mice. Exerc. Immunol. Rev. 16, 105-118 (2010)

39. K. Ohashi, J.L. Parker, N. Ouchi, A. Higuchi, J.A. Vita, N. Gokce, A.A. Pedersen, C. Kalthoff, S. Tullin, A. Sams, R. Summer, K. Walsh, Adiponectin promotes macrophage polarization toward an anti-inflammatory phenotype. J. Biol. Chem. 285(9), 6153-6160 (2010)

40. K. Li, W. Xu, Q. Guo, Z. Jiang, P. Wang, Y. Yue, S. Xiong, Differential macrophage polarization in male and female BALB/c - mice infected with coxsackievirus B3 defines susceptibility to viral myocarditis. Circ. Res. 105(4), 353-364 (2009)
41. D.G. Cook, M.A. Mendall, P.H. Whincup, I.M. Carey, L. Ballam, J.E. Morris, G.J. Miller, D.P. Strachan, C-reactive protein concentration in children: relationship to adiposity and other cardiovascular risk factors. Atherosclerosis 149(1), 139-150 (2000)

42. N.K. Vikram, A. Misra, M. Dwivedi, R. Sharma, R.M. Pandey, K. Luthra, A. Chatterjee, V. Dhingra, B.L. Jailkhani, K.K. Talwar, R. Guleria, Correlations of C-reactive protein levels with anthropometric profile, percentage of body fat and lipids in healthy adolescents and young adult in urban North India. Atherosclerosis 168(2), 305-313 (2003)

43. K. Nebeck, B. Gelaye, S. Lemma, Y. Berhane, T. Bekele, A Khali, Y. Haddis, M.A. Williams, Hematological parameters and metabolic syndrome: findings from an occupational cohort in Ethiopia. Diabetes Metab. Syndr. 6(1), 22-27 (2012)

44. H. Kaur, B. Adams-Huet, G. Smith, I. Jialal, Increased neutrophil count in nascent metabolic syndrome. Metab. Syndr. Relat. Disord. 11(2), 128-131 (2013)

45. B.J. Park, J.Y. Shim, H.R. Lee, D.H. Jung, J.H. Lee, Y.J. Lee, The relationship of platelet count, mean platelet volume with metabolic syndrome according to the criteria of the American Association of Clinical Endocrinologists: a focus on gender differences. Platelets 23(1), 45-50 (2012)

46. H. Pohjantahti-Maaroos, A. Palomaki, P. Kankkunen, R. Laitinen, S. Husgafvel, K. Oksanen, Circulating oxidized low-density lipoproteins and arterial elasticity: comparison between men with metabolic syndrome and physically active counterparts. Cardiovasc. Diabetol. 9, 41 (2010)

47. V. Sigurdardottir, B. Fagerberg, J. Hulthe, Circulating oxidized low-density lipoprotein (LDL) is associated with risk factors of the metabolic syndrome and LDL size in clinically healthy 58 -year-old men (AIR study). J. Intern. Med. 252(5), 440-447 (2002)

48. E. Coban, M. Ozdogan, G. Yazicioglu, F. Akcit, The mean platelet volume in patients with obesity. Int. J. Clin. Pract. 59(8), 981-982 (2005)

49. S.K. Nadar, A.D. Blann, S. Kamath, D.G. Beevers, G.Y. Lip, Platelet indexes in relation to target organ damage in high-risk hypertensive patients: a substudy of the Anglo-Scandinavian Cardiac Outcomes Trial (ASCOT). J. Am. Coll. Cardiol. 44(2), 415-422 (2004)

50. Y. Tavil, N. Sen, H.U. Yazici, F. Hizal, A. Abaci, A. Cengel, Mean platelet volume in patients with metabolic syndrome and its relationship with coronary artery disease. Thromb. Res. 120(2), 245-250 (2007)

\begin{tabular}{|l|lll|}
\hline Journal : Large 12020 & Dispatch : 19-9-2013 & Pages : 11 \\
Article No. : $\mathbf{6 7}$ & $\square$ LE & $\square$ TYPESET \\
MS Code : ENDO-D-13-00417 & $\sim_{\mathrm{CP}}$ & $\checkmark$ DISK \\
\hline
\end{tabular}


Journal : 12020

Article : 67

\section{Author Query Form}

\section{黛 Springer}

the language of science

\section{Please ensure you fill out your response to the queries raised below and return this form along with your corrections}

\section{Dear Author}

During the process of typesetting your article, the following queries have arisen. Please check your typeset proof carefully against the queries listed below and mark the necessary changes either directly on the proof/online grid or in the 'Author's response' area provided below

\begin{tabular}{|l|l|l|}
\hline Query & Details Required & Author's Response \\
\hline AQ1 & The reference [Jesri et al. [35]] seems to be mismatched. Please check and confirm. & \\
\hline AQ2 & Please update the reference [10] with vol. no. and page ranges. & \\
\hline
\end{tabular}

